\title{
CRECIMIENTO Y PRODUCCIÓN DE Donax obesulus REEVE, 1854 (BIVALVIA: DONACIDAE) EN PLAYA SARAPAMPA, ASIA, LIMA
}

\section{GROWTH AND PRODUCTION OF Donax obesulus REEVE, 1854 (BIVALVIA: DONACIDAE) AT SARAPAMPA BEACH, ASIA, LIMA}

\author{
Arturo Aguirre Velarde ${ }^{1}$ y Jaime Mendo Aguilar $^{1}$
}

\section{Resumen}

Se estimaron los parámetros de crecimiento y producción de la población de $D$. obesulus de la playa arenosa expuesta de Sarapampa, $100 \mathrm{~km}$ al sur de Lima. Para ello se realizaron muestreos mensuales durante 11 meses (Octubre 2005-Agosto 2006) en 6 estaciones distribuidas a lo largo de la playa. Se elaboraron frecuencias de tallas y se determinaron los parámetros de crecimiento de la ecuación de Von Bertalanffy (ECVB) mediante ELEFAN I. La tasa de mortalidad total (Z) se estimó por el método de la curva de captura linealizada. Los parámetros de crecimiento fueron $\mathrm{K}=$ $0.991 \mathrm{y} \mathrm{L}_{\infty}=33.70 \mathrm{~mm}$ y la tasa de mortalidad total $\mathrm{Z}=3.28$. La biomasa promedio fue estimada utilizando la relación talla-peso húmedo libre de valvas $\left(\mathrm{PHLV}=0.0003 \mathrm{~L}^{3.061}\right)$ en $54.10 \mathrm{~g}$ PHLV $\mathrm{m}^{-2}$. La producción de esta especie en términos de peso seco libre de ceniza (PSLC) se calculó en 3.76 g PSLC. $\mathrm{m}^{-2}$.año ${ }^{-1}$ en promedio. Los individuos de 22 y $23 \mathrm{~mm}$ de talla aportaron mayormente a la producción anual $(10.3 \%)$. La tasa de renovación anual $(P / \bar{B})$ fue estimada en 1.20 . Se discute el crecimiento y la productividad de esta especie en relación a otras del mismo género.

Palabras clave: Donax obesulus, crecimiento, producción, biomasa, playas arenosas, Perú

\begin{abstract}
Growth parameters and production of the population of $D$. obesulus at the exposed sandy beach of Sarapampa (100 km south of Lima) were estimated. Monthly samplings were carried out during a year (October 2005-August 2006) in 6 stations distributed throughout the beach. Lengthfrequency distributions were elaborated and the growth parameters of the Von Bertalanffy growth equation (VBGE) determined by ELEFAN I. Total mortality rate (Z) was estimated by the lengthbased catch curve method and production of somatic and shell components were estimated by the mass specific growth rate method. Growth parameters were estimated as $\mathrm{K}=0.991$ and $\mathrm{L}_{\infty}=33.70$ $\mathrm{mm}$ and total mortality as $\mathrm{Z}=3.28$. Mean biomass was calculated using the relation length-shell and free wet mass $\left(\mathrm{SFWM}=0.0003 \mathrm{~L}^{3.061}\right.$ ) as $54.10 \mathrm{~g} \mathrm{SFWM} \cdot \mathrm{m}^{-2}$. The average production of this species was calculated in terms of ash free dry weight in $3.76 \mathrm{~g}$ AFDW. $\mathrm{m}^{-2}$.year ${ }^{-1}$. Individuals ranging from 22 to $23 \mathrm{~mm}$ contributed most to the total production (10.3\%). Mean annual turnover rate $(P / \bar{B})$ was 1.20 . Growth and production of this species is compared with other species of the same genus.
\end{abstract}

Key words: Donax obesulus, growth, production, biomass, sandy beaches, Peru

\section{Introducción}

Donax obesulus es un bivalvo perteneciente a la familia Donacidae que tiene una amplia distribución en la costa peruana en donde es conocida comúnmente como "palabrita" o "concha blanca" (Paredes \& Cardoso, 2001). Como es característico de la familia Donacidae, habitan en playas arenosas expuestas donde se alimentan principalmente de fitoplancton y detritus orgánico en suspensión (Mori, 1938; Ansell \& Trevallion, 1969; Penchaszadeh \& Oliver, 1975; Carchi, 1978; Ansell, 1983). Las especies del género Donax pueden dominar las comunidades de macrozoobentos en número y en biomasa (McLachlan et al., 1981; Arntz \& Fahrbach, 1991; Ieno \& Bastida, 1998), jugando un papel ecológico importante en el acoplamiento bento-pelágico (Wade, 1968; McLachlan et al., 1981; McLachlan \& Lewin, 1981; McDermontt, 1983; DeLancey, 1989). Donax obesulus se distribuye desde Canoa, Manabí, Ecuador hasta La Rinconada, Bahía Moreno, Antofagasta, Chile (Coan, 1983). En Perú, Paredes \& Cardoso (2001) destacan los bancos de Tumbes, Piura, Lambayeque, Ancash y Lima, sin embargo, en la actualidad los bancos de la zona sur de Perú han cobrado importancia debido a su explotación con fines de exportación, principalmente hacia mercados europeos. Según el Ministerio de Producción del Perú se exportaron $2650 \mathrm{TM}$ de esta especie durante el año 2005 a nivel nacional. 
Uno de los bancos de Donax obesulus más abundantes y que hasta ahora permanece inexplotado en la zona de Lima se ubica en Playa Sarapampa. A pesar de la gran importancia ecológica y económica de esta especie en la zona, no existen estudios realizados sobre su biología, ecología y dinámica poblacional. Solo existen algunos estudios realizados en otras zonas del litoral peruano relacionados con su taxonomía (Paredes \& Cardoso, 2001), crecimiento (Arntz et al., 1987), ecotoxicología (Lacma et al., 2007) y parasitología (Pérez-Cordón et al., 2007). Es por ello que este estudio, con el fin de aportar al conocimiento de esta especie, evalúa aspectos básicos de la dinámica poblacional de este banco que permitan sentar las bases para su futuro manejo y aprovechamiento sostenible.

\section{Materiales y Métodos}

\section{Lugar del estudio}

Este estudio se realizó en la Playa Sarapampa, ubicada al Sur de Lima en el distrito de Asia a la altura del Kilómetro 109 de la carretera Panamericana Sur entre los $12^{\circ} 49^{\prime} 18.8^{\prime}$ 'S y $12^{\circ} 51^{\prime} 5.96^{\prime} \mathrm{S}$ (Figura 1). Esta playa es amplia y arenosa en toda su extensión con una pendiente suave y fuerte oleaje. La distribución de $D$. obesulus en el área de estudio esta limitada por zonas rocosas en la parte Norte de la playa y de acantilados en la parte Sur, con una extensión de $5520 \mathrm{~m}$.

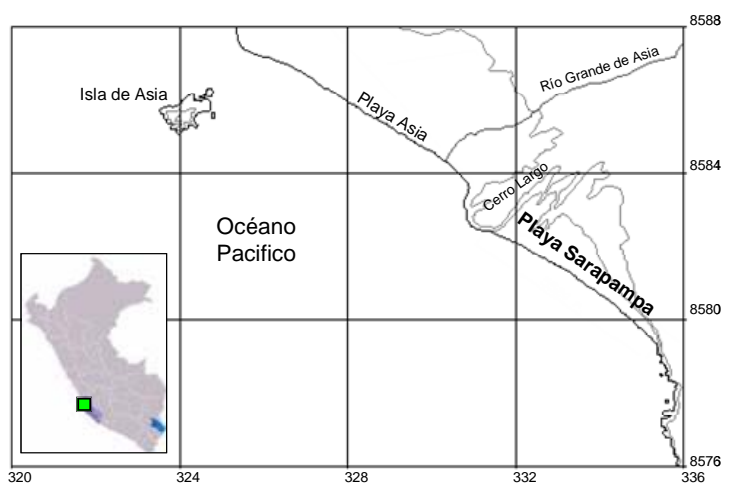

Figura 1. Ubicación de Playa Sarapampa.

Estimación de parámetros de crecimiento, mortalidad y reclutamiento.

Para la estimación de los parámetros de crecimiento y mortalidad se colectaron mensualmente individuos de $D$. obesulus en 6 estaciones de muestreo a lo largo de la playa durante Octubre 2005 hasta Agosto 2006. En cada estación se barrió una extensión de $50 \mathrm{~m}$ de línea costera entre los 0.20 y $1.5 \mathrm{~m}$ de profundidad para tener representatividad en las tallas. Se colectaron entre 500 y 1000 individuos en cada muestreo. La extracción de los individuos se realizó manualmente utilizando una red de captura tipo bolsa con un tamaño de malla de $2 \mathrm{~mm}$ donde se colocó y tamizó la arena colectada de hasta $20 \mathrm{~cm}$ del fondo. De cada individuo se registro la longitud anteroposterior de la valva con un vernier de $0.05 \mathrm{~mm}$ de precisión. Se utilizó la ecuación de Von Bertalanffy (ECVF) estacionalizada (Cloern \& Nichols, 1978) para ajustar la curva de crecimiento:

$$
\mathrm{L}_{\mathrm{t}}=\mathrm{L}_{\infty}\left[1-\mathrm{e}^{\left(-\mathrm{K}^{*}(\mathrm{t}-\mathrm{to})-(\mathrm{CK} / 2 \pi)^{*} \operatorname{sen}\left(2 \pi^{*}(\mathrm{t}-\mathrm{SP})\right)\right)}\right]
$$

En donde $\mathrm{L}_{\mathrm{t}}$ es la talla correspondiente a la edad $\mathrm{t}$, $\mathrm{L}_{\infty}$ la longitud asintótica, $\mathrm{K}$ el parámetro de curvatura, $\mathrm{t}_{\mathrm{o}}$ la edad a la longitud cero, $\mathrm{C}$ la amplitud de oscilación y $\mathrm{SP}$ el punto de verano $(\mathrm{WP}=\mathrm{SP}+0.5$, punto de invierno). Los parámetros de crecimiento fueron estimados con el programa FISAT II (Fish Stock Assessment Tools) usando las frecuencias de tallas con la rutina de ELEFAN I (Electronic Length Frequency Análisis). Adicionalmente se determinó el valor de $\mathrm{t}_{0}$, usando la ecuación de Pauly (1980):

$$
\log \left(-\mathrm{t}_{0}\right)=-0.3922-0.2752 \cdot \log \left(\mathrm{L}_{\infty}\right)-1.038 \cdot \log (\mathrm{K})
$$

Con los datos recolectados de los muestreos acumulados se elaboro la curva de captura linealizada con el objetivo de hallar la tasa de mortalidad $(Z)$ del modelo de extinción exponencial:

$$
\mathrm{Nt}=\mathrm{Nt}-1\left(\mathrm{e}-\mathrm{Z}^{*} \Delta \mathrm{t}\right)
$$

En este caso asumimos $Z=M$ ya que en esta playa no existe extracción de esta especie.

El tiempo de vida $\left(\mathrm{t}_{\max }\right)$ teórico para la población de $D$. obesulus en playa Sarapampa fue estimado mediante la inversa de la ecuación de crecimiento de Von Bertalanffy (Taylor, 1958):

$$
\mathrm{t}_{\max }=\left[\ln \mathrm{L}_{95 \%}-\ln \left(\mathrm{L}_{\infty}-\mathrm{L}_{95 \%}\right)\right] / \mathrm{K}
$$

Donde $\mathrm{L}_{95 \%}$ representa el $95 \%$ de la longitud antero-posterior máxima registrada durante los muestreos.

Para comparar los parámetros de crecimiento con otras especies de Donax se calculó la tasa de crecimiento en el punto de inflexión $(\mathrm{P})$ de la curva talla-peso (Pauly, 1979) y el índice de rendimiento en crecimiento $\Phi^{\prime}$ (Pauly \& Munro, 1984):

$$
\begin{gathered}
\mathrm{P}=\log \left[\mathrm{K}\left(\mathrm{L}_{\infty}\right)^{3}\right] \\
\Phi^{\prime}=2 \log \left(\mathrm{L}_{\infty}\right)+\log (\mathrm{K})
\end{gathered}
$$

Finalmente se determinó el patrón de reclutamiento de esta especie con la rutina de FISAT usando las frecuencias de tallas y los parámetros de crecimiento de acuerdo a Pauly (1982).

Densidad, biomasa y producción.

La densidad y biomasa de $D$. obesulus por tallas fueron estimadas mediante el método de área barrida 
(Bazigos, 1980; Espino \& Wosnitza-Mendo, 1984; Sparre \& Venema, 1997). El área de distribución de esta especie en la zona de estudio fue dividida en 2 estratos de acuerdo al tipo de sustrato (arenoso y arena con conchuela). Un total de 24 estaciones fueron realizadas y distribuidas en cada estrato mediante locación óptima o de Neyman (Sparre \& Venema, 1997). El muestreo en cada estación se realizó con un cilindro plástico de $0.196 \mathrm{~m}^{2}$ de área y de $20 \mathrm{~cm}$ de profundidad, el cual fue enterrado en el fondo. El contenido total del cilindro fue extraído e introducido en una malla de $1 \mathrm{~mm}$ de tamaño de malla y los individuos de $D$. obesulus fueron contabilizados y medidos (longitud total, L) con un vernier de $0.05 \mathrm{~mm}$ de precisión.

Un total de 400 individuos fueron separados de manera estratificada por tallas, medidos y pesados (peso total, PT) con una balanza de $0.1 \mathrm{~g}$ de precisión, para la determinación de los parámetros de la relación talla-peso total. De igual manera fueron separados 80 individuos para la obtención de los parámetros de la relación talla-peso húmedo libre de valvas (PHLV). En ambos casos se uso la relación potencial $\mathrm{W}=\mathrm{a}^{*} \mathrm{~L}^{\mathrm{b}}$.

La producción de la población $P$ (en gramos de PHLV por metro cuadrado por año) fue calculada con el método de la tasa de crecimiento específico en peso (Crisp, 1984; Brey, 2001) utilizando los datos de densidad, frecuencia de longitud, los parámetros de la ECVB y las relaciones longitud-peso. Según la formula:

Donde,

$$
P_{\text {PHLV }}=\sum \mathrm{N}_{\mathrm{i}} * \mathrm{M}_{\mathrm{i}} * \mathrm{G}_{\mathrm{i}}\left[\mathrm{g} \text { PHLV } \cdot \mathrm{m}^{-2} \cdot \mathrm{año}^{-1}\right]
$$

$\mathrm{N}_{\mathrm{i}}$ : Numero de individuos $/ \mathrm{m}^{2}$

$\mathrm{M}_{\mathrm{i}}$ : Peso individual de tejido húmedo libre de valvas de la clase $\mathrm{i}$

$\mathrm{G}_{\mathrm{i}}$ : Tasa de crecimiento especifico:

$\mathrm{G}_{\mathrm{i}}=\mathrm{b}_{(\mathrm{PHLV})} * \mathrm{~K}^{*}\left(\left(\mathrm{~L}_{\infty} / \mathrm{L}_{\mathrm{i}}\right)-1\right)\left[\mathrm{año}{ }^{-1}\right]$

Se calculó la producción individual $P_{\mathrm{PHLV}}=\mathrm{Mi}{ }^{*} \mathrm{Gi}$ en $\mathrm{g}$ PHLV. año ${ }^{-1}$ y la producción de valvas $P_{\mathrm{V}}=$ $\left(\mathrm{M}_{\mathrm{PTi}}{ }^{*} \mathrm{G}_{\mathrm{PTi}}\right)-P_{\text {ind }}$, donde $\mathrm{M}_{\mathrm{PTi}}$ es el peso total individual de la clase i y $\mathrm{G}_{\mathrm{PTi}}$ es la tasa de crecimiento especifico en peso total $\left(\mathrm{G}_{\mathrm{PTi}}=\mathrm{b}_{(\mathrm{PT})} * \mathrm{~K}^{*}\left(\left(\mathrm{~L}_{\infty} / \mathrm{L}_{\mathrm{i}}\right)-1\right)\left[\mathrm{año}^{-1}\right]\right.$.

Se calculó la tasa de renovación anual $(P / \bar{B})$ a partir de la producción anual y de la biomasa media anual obtenida con $\bar{B}=\mathrm{N}_{\mathrm{i}}{ }^{*} \mathrm{PHLV}_{\mathrm{i}}$. Con el fin de comparar los resultados de esta especie con otros estudios y otras especies del mismo género, los datos de producción y tasa de renovación anual fueron convertidos a peso seco libre de ceniza (PSLC), usando el factor de conversión PSLC/PHLV de 5.8\% (Ricciardi \& Bourget, 1998).

\section{Resultados}

Crecimiento, mortalidad y reclutamiento

Durante el periodo de estudio se recolectaron y midieron 9943 individuos de $D$. obesulus. La talla máxima registrada fue de $31.0 \mathrm{~mm}$ y la mínima de 5.4 $\mathrm{mm}$. En la Figura 2 se muestran las frecuencias de tallas obtenidas en cada muestreo y el ajuste de la curva de la ECVB con los parámetros obtenidos en ELEFAN I. Se observó la presencia de 3 cohortes. El primer cohorte estuvo presente en los muestreos desde octubre 2005 hasta Noviembre del mismo año, el segundo cohorte desde octubre 2005 hasta Julio del 2006, y el tercer cohorte fue reclutado en Marzo del 2006 y estuvo presente hasta el fin de los muestreos. Los parámetros de crecimiento fueron estimados en $\mathrm{K}=0.991, \mathrm{~L}_{\infty}=33.7, \mathrm{t}_{0}=-0.12, \mathrm{C}=0.50, \mathrm{WP}=0.70$. Los índices de crecimiento $\Phi^{\prime}$ y $\mathrm{P}$ tuvieron valores de 3.051 y 4.579 , respectivamente.

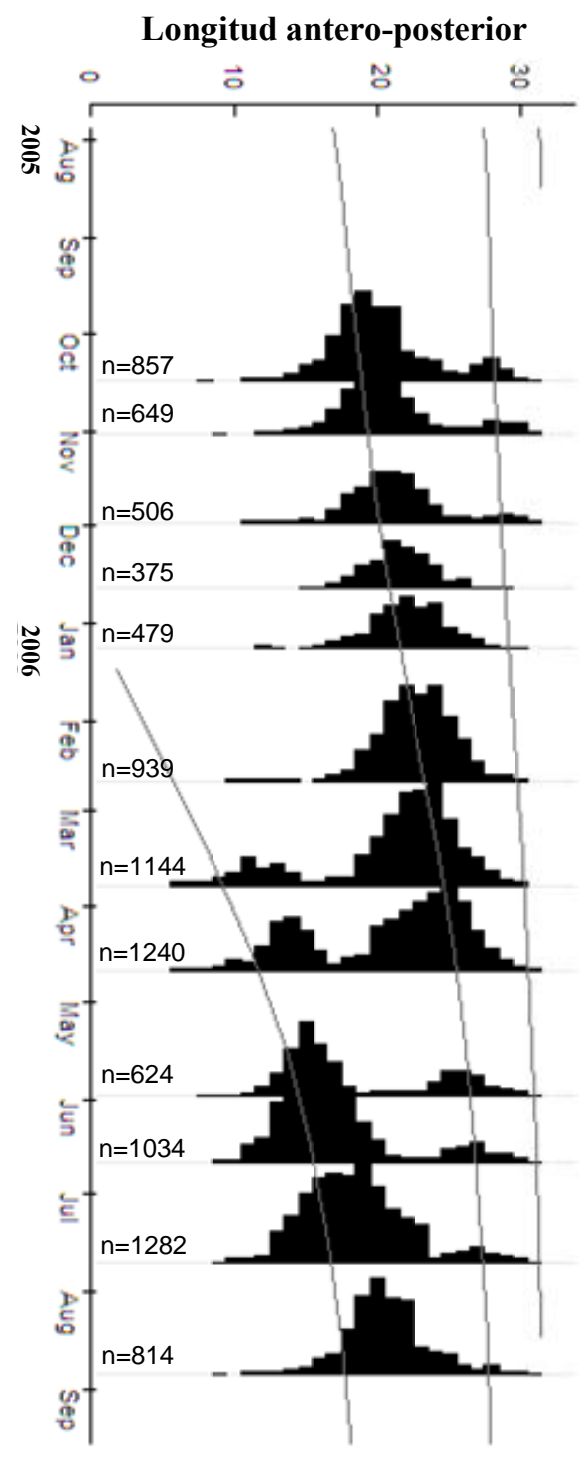

Figura 2. Frecuencia de tallas de Donax obesulus durante el tiempo de estudio. 
Según el patrón de reclutamiento obtenido (Figura 3), D. obesulus se recluta una vez al año y con mayor intensidad durante los meses de Abril y Mayo.

La tasa de mortalidad total de Z calculada por medio de la curva de captura linealizada (Figura 4) fue de 3.28 , utilizando la captura acumulada durante todo el periodo de estudio.

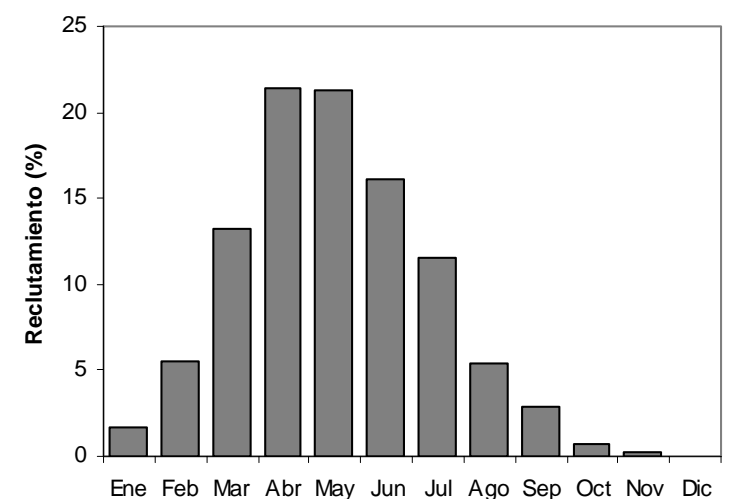

Meses

Figura 3. Patrón de reclutamiento de D. obesulus.

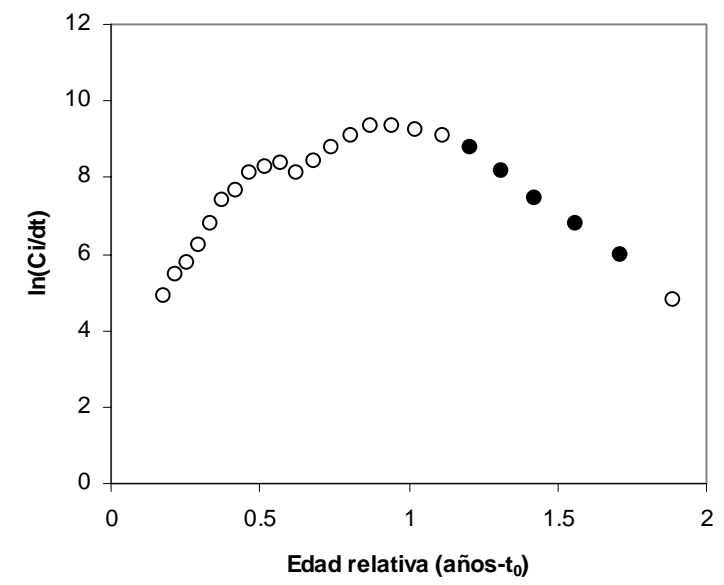

Figura 4. Curva de captura linealizada. En negro los puntos utilizados en la regresión.

\section{Biomasa y producción somática}

La relación entre la longitud antero-posterior y el peso total para la población de $D$. obesulus en la playa Sarapampa es expresada por la ecuación PT $=0.0004$ $\mathrm{L}^{2.7146}\left(\mathrm{R}^{2}=0.9208\right)$ y la relación entre la longitud antero posterior y el peso húmedo libre de valvas esta expresada por la ecuación PHLV $=0.0003 \mathrm{~L}^{3.061}$ $\left(\mathrm{R}^{2}=0.9210\right)$.

Las densidades registradas en el área de estudio variaron entre 472.8 y 6.5 ind $\mathrm{m}^{-2}$ con un promedio de 117.1 ind $\mathrm{m}^{-2}$. En la Tabla 1 se muestran las densidades por estratos así como las respectivas biomasas encontradas. Las mayores densidades fueron registradas en la zona centro-sur. La biomasa total de esta especie en el área de estudio fue estimada en $53173.178 \mathrm{~kg}$ PT y en $11945.2 \mathrm{~kg}$ PHLV con un promedio de $54.1 \mathrm{~g}$ PHLV. $\mathrm{m}^{-2}$. Espacialmente $D$. obesulus se distribuyó principalmente en la zona central y sur de la playa donde predomina el estrato arenoso de 20-150 cm alcanzando altas densidades.

La producción total de tejidos blandos de $D$. obesulus $(P)$ fue estimada en $64.85 \mathrm{~g}$ PHLV.m ${ }^{-2}$.año ${ }^{-1}$. La producción individual muestra que los individuos de $22 \mathrm{~mm}$ de longitud tienen una mayor producción de tejido blando con respecto a otras tallas con 0.62 g.año ${ }^{-1}$ y que la mayor producción en peso de valvas se da a los $20 \mathrm{~mm}$ con 1.91 g.año $^{-1}$ (Figura 5). Los individuos entre 22 y $23 \mathrm{~mm}$ aportan una mayor producción a la población de Playa Sarapampa con respecto a otra tallas del rango, representando el $10.27 \%$ sobre el total (Figura 6). En términos de peso seco libre de ceniza utilizando el factor de conversión de Ricciardi \& Bourget (1998), la producción $(P)$ fue de $3.76 \mathrm{~g}$ PSLC. $\mathrm{m}^{-2}$.año ${ }^{-1}$ y la biomasa promedio $(\bar{B})$ fue $3.14 \mathrm{~g}$ PSLC.m ${ }^{-2}$. La tasa de renovación anual $(P / \bar{B})$ calculada fue de 1.20 .

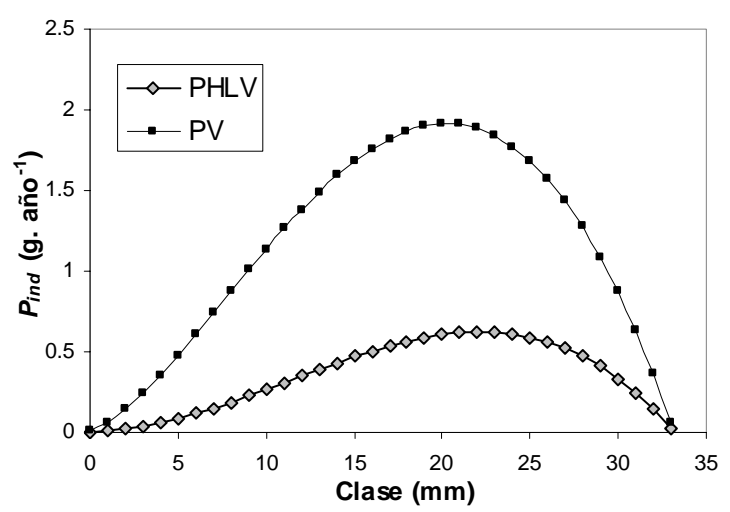

Figura 5. Producción individual de D. obesulus en valvas $\left(\mathrm{P}_{\mathrm{V}}\right)$ y Peso húmedo libre de valvas $\left(P_{\mathrm{PHLV}}\right)$.

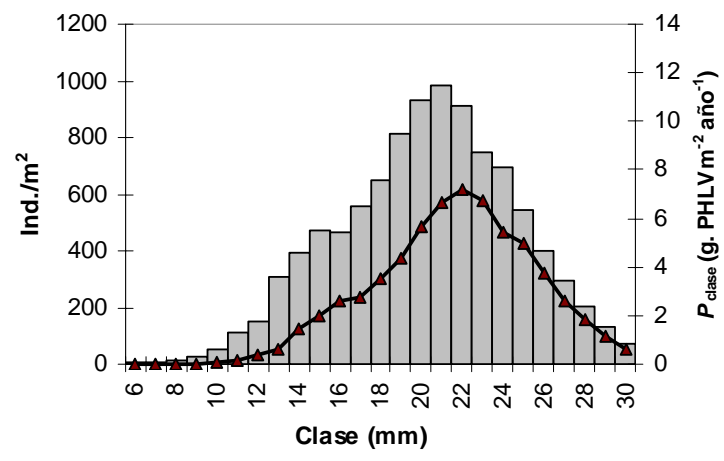

Figura 6. Producción y densidad de D. obesulus por clase de talla. 


\section{Discusión \\ Crecimiento y reclutamiento}

Arntz et al., (1987) estudiaron la población de $D$. obesulus de Playa Santa María ubicada al sur de Lima (a $53 \mathrm{~km}$ al norte de Playa Sarapampa) antes, durante y después del Evento El Niño del año 1982. Los valores reportados por este autor utilizando ELEFAN fueron de $K=1.00$ y $_{\infty}=46.00$ (previo El Niño), $K=$ 1.17 y $\mathrm{L}_{\infty}=35.00$ (durante El Niño) y $\mathrm{K}=0.70 \mathrm{y} \mathrm{L}_{\infty}=$ 42.00 (posterior El Niño). Los parámetros estimados en este estudio son similares a los encontrados por Arntz et al., (1987) durante El Niño del año 1982, sin embargo el valor de $\Phi^{\prime}$ calculado en este estudio es similar al obtenido por estos autores para después de El Niño (Tabla 3). Ello se puede deber a que los valores, en especial de $\mathrm{L}_{\infty}$ publicados por Arntz et al., (1987), exceden en gran magnitud a la talla máxima registrada en este y otros estudios para $D$. obesulus (<33 mm) (Guzmán et al., 1998; Paredes \& Cardoso, 2001). Sin embargo, no se descarta la posibilidad de que la talla máxima pueda estar subestimada, considerando que, el muestreo de esta especie se realiza hasta profundidades menores de $1.5 \mathrm{~m}$. En cuanto a los parámetros de estacionalidad del crecimiento, los valores de WP son muy similares a los encontrados por Arntz et al. (1987) (0.60-0.67). En contraste, el $\mathrm{C}$ obtenido en el presente estudio es mucho mas bajo que los encontrados por Arntz et al. (1987) (>0.98). El valor estimado en este estudio se ajusta mejor a las diferencias de temperatura que se registran en verano e invierno en las playas del sur de Lima $\left(5^{\circ} \mathrm{C}\right)$. El índice de rendimiento en crecimiento $\mathrm{P}$ calculado para $D$. obesulus en Playa Sarapampa es ligeramente alto en comparación con el de otras especies del género, en especial con relación a las de $\mathrm{L}_{\infty}$ similares, debido probablemente a la alta productividad que caracteriza a las costas del Perú (Tabla 2). Sin embargo, cabe mencionar que este valor de $\mathrm{P}$ se aleja notoriamente del valor publicado por Arntz et al. (1987) para el periodo antes de El Niño 1983. La posibilidad de una sobre estimación de los valores de $\mathrm{L}_{\infty}$ ó $\mathrm{K}$ publicados por estos autores podría ser sustentado en base al valor de $\mathrm{t}_{\max }=0.58$ años estimado de acuerdo a Taylor, (1958), muy por debajo de la edad real del cohorte mostrada en las frecuencias

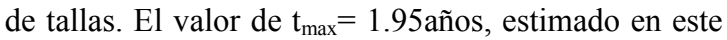
estudio, concuerda con McLachlan, (1979) y Ansell, (1983) quienes sostienen que la mayoría de especies del género Donax tienen un tiempo de vida relativamente corto, entre 1 y 2 años. El pico de reclutamiento observado durante el mes de Abril, concuerda con lo encontrado con Arntz et al. (1987) durante el año previo y posterior a El Niño quienes reportan el reclutamiento durante los primeros meses del año.

Densidad, Biomasa y producción

Las densidades encontradas en Playa Sarapampa son relativamente altas con respecto a las encontradas por Arntz et al., (1987) en playa Santa Maria. La mayor competencia por espacio y alimento con las especies Mesodesma donacium y Emerita analoga en Playa Santa María que en Playa Sarapampa donde solo habita Emerita analoga, explicaría en parte estas diferencias en densidad. Por otro lado el hecho que Playa Santa María se encuentre mas urbanizada y reciba una mayor cantidad de turistas que Playa Sarapampa podría significar un mayor impacto de tipo antropogénico sobre el asentamiento y reclutamiento de esta especie. Al respecto Hermman et al., (en prensa) encontraron un posible efecto del turismo durante los meses de verano en las densidades de $D$. hanleyanus. No existen trabajos sobre biomasa $\mathrm{y}$ producción de D. obesulus en Perú y en otras regiones, sin embargo la información existente al respecto de otras especies del genero Donax muestran una producción y biomasa relativamente alta de esta especie (Tabla 3 ) con excepcion de $D$. serra y $D$. trunculus que tienen una producción mucho mayor debido a que alcanzan una mayor talla y peso individual. Sin embargo la tasa de renovación anual $(P / \bar{B})$ de Donax obesulus en el presente estudio es comparable a la de $D$. serra, ambas especies propias de zonas de afloramiento.

\section{Conclusiones}

Los parámetros de crecimiento $D$. obesulus concuerdan con los reportados para otras especies del genero Donax. Es una especie que muestra estacionalidad en su crecimiento mostrando una oscilación moderada

D. Obesulus en Sarapampa presenta altas densidades (117.1 ind $/ \mathrm{m}^{2}$ ) y biomasas (3.14 g PSLC $\mathrm{m}^{-2}$ ) con una alta producción (3.76 $\mathrm{g}^{\text {PSLC m}} \mathrm{m}^{-2}$ año $^{-1}$ ) en comparación con otras especies del género, en especial a las de $\mathrm{L}_{\infty}$ similar.

La población de D. obesulus en Sarapampa se recluta una vez al año, con en mayor intensidad durante los meses de Marzo-Abril.

\section{Agradecimientos}

Agradecemos a la Ing. Vanessa Quevedo Alvarado y Diana Aranda Pariasca por su franca amistad y su comprometido apoyo durante la realización de este trabajo, el cual no hubiera sido posible sin su cooperación.

\section{Literatura citada}

Ansell A.D. 1983. The biology of genus Donax. In: McLachlan A., T. Erasmus \& W. Junk. (Eds.) Developments in Hydrobioly Vol. 19. Sandy Beaches as ecosystem. Dr. W. Junk Publishers, The Hague, The Netherlands, The Netherlands.: 607-635.

Ansell A.D. \& Trevallion A. 1969. Behavioural adaptations of intertidal molluscs from a tropical sandy beach. J. exp .mar. Biol. Ecol. 4: 9-35.

Arntz W.E., Brey T., Tarazona J. \& Robles A. 1987. Changes in the Structure of a Shallow sandy-beach 
community in Perú during an El Niño event. South Africa Journal of Marine Science. 5: 645-658.

Arntz W.E. \& Fahrbach E. 1991. El Niño Klimaexperiment der Natur: Die physikalischen ursachen und biologischen folgen. Birkhäuser, Basel, Bostem, Berlin.

Ansell A.D. \& Lagardère F. 1980. observations on the biology of Donax trunculus and Donax vittatus at Ile d'Oleron (French Atlantic Coast). Marine Biology. 57(4): 287-300.

Bazigos G.P. 1980. El diseño de reconocimientos de pesca con redes de arrastre. FAO, COCIC IX/11.

Brey T. 2001. Population dynamics in benthic invertebrates. A virtual handbook. Alfred Wegener Institute for Polar and Marine Research, Germany.

Carchi W. 1978. Functional anatomy of Donax hanleyanus Philippi 1847 (Donacidae-Bivalvia). Boletim de Zoologia/Departamento de Zoologia, Instituto de Biociências, Universidade de Sao Paulo. 3: 121-142.

Cardoso R.S. \& Veloso V.G. 2003. Population dynamics and secondary produccion of the wedge clam Donax hanleyanus (Bivalvia: Donacidae) on a high-energy, sub-tropical beach of brazil. Marine Ecology. 142: 153162.

Cloern J.E. \& Nichols F.H. 1978. A von Bertalanffy growth model with a seasonally varying coefficient. J. Fish. Res. Board. Can. 35: 1479-1484.

Coan E. V. 1983. The Eastern Pacific Donacidae. The Veliger. 25(4): 273-298.

Crisp D.J. 1984. Energy flow measurements. En: Holme, N.A. \& McIntyre, A.D. (Eds.) Methods for the study of marine benthos. Blackwell Scientific Publications, Oxford, IBT Handbook no. 16: 284-371.

Defeo O. 1996. Recruitment variability in sandy beach macroinfauna: much to learn yet. Revista Chilena de Historia Natural. 69: 615-630.

DeLancey L.B. 1989. Trophic relationship in the surf zone during the summer at Folly Beach, South Carolina. Journal of Coastal Research. 5: 477-488.

Espino M. \& Wosnitza-Mendo C. 1984. Manuales de evaluación de peces $\mathrm{N}^{\circ} 1$. Area Barrida. Inf. Inst. Mar Perú, 86.

García N., Prieto A., Alzola R. \& Lodeiros C. 2003. Crecimiento y distribucion de tallas de Donax denticulatus (Mollusca: Donacidae) en playa Brava, Peninsula de Araya, estado Sucre, Venezuela. Revista cientifica / Universidad del Zulia. 13: 464-470.

Gianuca N.M. 1985. The ecology of sandy beach in Soutern Brazil. DSc Thesis, University of Southamton.

Guzmán N., Saá S. \& Ortlieb L. 1998. Catálogo descriptivo de los moluscos litorales (Gastropoda y Pelecypoda) de la zona de Antofagasta, $23^{\circ} \mathrm{S}$ (Chile).

Hermman M., Laudien J., Penchaszadeh P.E., Fischer S. \& Arntz W. In Press. Population structure and produccion of the surf clam Donax hanleyanus (Bivalvia: Donacidae) from norther Argentinean beaches.

Ieno E.N. \& Bastida R.O. 1998. Spatial and temporal patterns in coastal macrobenthos of Samborombon Bay, Argentina: a case study of very low diversity. Estuarios. 21 (4B): 690-699.

Lacma J., Iannacone J. \&. Giovanna V. 2007. Toxicidad del Cromo en sedimento usando Donax obesulus Reeve 1854 (Pelecypoda: Donacidae) Ecología Aplicada. 6(1,2):93-99.
Laudien J., Brey T. \& Arntz W.E. 2003. Population structure, growth and production of the surf clam Donax serra (Bivalvia: Donacidae) on two Namibian sandy beaches. Estuarine, Coastal and Shelf Scince. 58S: 105115.

Luzzatto D.C. 2007. Dinámica poblacional de la almeja amarilla (Mesodesma mactroides) y del berberecho (Donax hanleyanus) en intermareales de la playas arenosas del nordeste de la provincia de Buenos Aires. Ph.D. Thesis, Universidad de Buenos Aires, Facultad de Ciencias Exactas y Naturales.

Marcano J.S., Prieto A., Lárez A. \& Salazar H. 2003. Crecimiento de Donax denticulatus (Linné 1758) (Bivalvia. Donacidae) en la ensenada La Guardia, isla de Margarita, Venezuela. Zootecnia tropical. 21(3): $237-$ 259.

McDermontt J.J. 1983. Food web in the surf zone of an exposed sandy beach along the mid-Atlantic coast of the United States. En: McLachlan A., T. Erasmus \& W. Junk. (Eds.) Sandy Beaches as ecosystem. Dr. W. Junk Publishers, The Hague, The Netherlands.: 529-538.

McLachlan A. 1979. Growth and production of Donax sordidus Hanley on an open sandy beach in Algoa Bay. South African Journal of Zoology. 14: 61-66.

McLachlan A., Erasmus T., Dye A.H., Woolridge T., Van der Horst G., Rossouw G., Lasiak T.A. \& McGwynne L.E. 1981. Sand beach energetics: an ecosystem approach towards a high energy interface. Estuarine, Coastal and Shelf Science. 13: 11-25.

McLachan A. \& Lewin J. 1981. Observations on surf phytoplankton blooms along the coasts of South Africa. Botanica Marina .24 (10): 553-557.

McLachlan A. \& Van der Horst G. 1979. Growth and production of two molluscs from an exposed sandy beach. South African Journal of Zoology. 14: 194-201.

Mori S. 1938. Characteristic tidal rhythmic migration of mussel, Donax semignosus Dunker, and the experimental analysis of behavior at the flood tide. Zoological Magazine Tokio. 50: 1-12.

Paredes C. \& Cardoso F. 2001. El Género Donax en la Costa Peruana (Bivalvia: Tellinoidea). Revista Peruana de Biología. 8(2):83-93.

Palacios J., Cruz R. \& Pacheco O. 1983. Estructura poblacional y cuantificación de Donax dentifer Hanley 1843.

Pauly D. 1979. Gill size and temperature as governing factors in fish growth: a generalization of von Bertalanffy's growth formula. Berichte des Institut für Meereskunde an der Universität Kiel. No. 63, xv +156 p.

Pauly D. 1980. A new methodology for rapidly acquiring basic information on tropical fish stocks: growth, mortality and stock-recruitment relationships, p. 154 172. In: S. Saila \& P. Roedel (Eds.) Stock Assessment for Tropical Small-Scale Fisheries. Proc. Intern. Workshop, Sept. 19-21 (1979) Univ. Rhode Island. Intern. Center Mar. Resource Development.

Pauly D. 1982. Une sélection de méthodes simples pour l'estimation des stocks de poissons tropicaux. FAO Circulaire sur les pêches $\mathrm{N}^{\circ} 729$.

Pauly D. \& Munro J.L. 1984. Once more on the comparison of growth in fish and invertebrates. ICLARM Fishbyte. 2(1): 21 . 
Ecol. apl. Vol. $7 \mathrm{~N}^{\mathrm{os}} 1$ y 2 , pp. 63-70

Penchaszadeh P.E. \& Oliver S.R. 1975. Ecología de una población de "berberecho" (Donax hanleyanus) en Villa Gesell, Argentina. Malacología. 15: 133-146.

Perez-Cordon G., Rosales M. \& Gavira J. 2007. Hallazgo de Blastocystis sp. en bivalvos del género Donax. Rev. peru biol.14(2): 301-302.

Ricciardi A. \& Bourget T.E. 1998. Weight-to-weight convesion factors for marine benthic macroinvertebrates. Marine Ecology Progress Series. 163: 245-251.

Riascos J.M. \& Urban H.J. 2002. Dinámica poblacional de Donax dentifer (Veneroida: Donacidae) en Bahía Málaga, Pacífico Colombiano durante el fenómeno El Niño 1997/1998. Revista de Biología Tropical. 50(3/4): 1113-1123.

Rocha-Barreira de Almeida C., Batista W.F., Monteiro D.O. \& Frankling-Junior W. 2002. Aspectos da estrutura populacional de Donax striatus (Linnaeus 1758)
(Mollusca: Donacidae) na praia do Futuro, Fortaleza CE. Arquivos de Ciencias do Mar. 35: 51-55.

Sparre P. \& Venema S.C. 1997. Introducción a la evaluación de recursos pesqueros tropicales. Parte 1. Manual. FAO Fish. Tech. Pap., No306.1, Rev. 2. Roma, FAO.

Taylor C.C. 1958. Cod growth and temperature. Journal du Conseil Permanent International pour 1'Explration de la Mer. 23: 266-370.

Wade B.A. 1968. Studies on the biology of the West Indian beach clam, Donax denticulatus L.2. Life history. Bulletin of Marine Science. 17: 723- 740.

Warwick R.M., C.L. George \& J.R. Davies 1987. Annual macrofauna production in a Venus community. Journal of the Marine Biological Association of the United Kingdom. 55: 1-18.

\section{Tablas citadas en el texto}

Tabla 1. Densidades y biomasas de D. obesulus para cada estrato de muestreo en Playa Sarapampa.

\begin{tabular}{|c|c|c|c|c|c|c|c|c|}
\hline \multirow[b]{2}{*}{ Estrato } & \multicolumn{4}{|c|}{ Densidad } & \multicolumn{4}{|c|}{ Biomasa } \\
\hline & $\begin{array}{l}\text { Area } \\
\left(\mathrm{m}^{2}\right)\end{array}$ & Ind. $/ \mathbf{m}^{2}$ & sd. & Limit. Conf. & g PT $/ \mathrm{m}^{2}$ & $\begin{array}{c}\text { Biomasa } \\
\text { Kg PT }\end{array}$ & G PHLV / $\mathbf{m}^{2}$ & $\begin{array}{r}\text { Biomasa } \\
\text { Kg PHLV }\end{array}$ \\
\hline Arenoso & 50750 & 472.8 & 282.71 & $315.9-629.7$ & 972.67 & 49363.228 & 218.509 & 11089.310 \\
\hline \multirow[t]{2}{*}{ Areno-conchueloso } & 170050 & 10.9 & 4.95 & $7.1-15.8$ & 22.40 & 3809.950 & 5.033 & 855.895 \\
\hline & & & & & Total $_{\mathrm{PT}}=$ & 53173.178 & Total $_{\mathrm{PHLV}}=$ & 11945.204 \\
\hline
\end{tabular}

Tabla 2. Parámetros de crecimiento de especies del género Donax en distintas partes del mundo.

\begin{tabular}{lcccccl}
\hline \multicolumn{1}{c}{ Especie } & País & $\mathbf{K}$ & $\mathbf{L}_{\infty}$ & $\mathbf{\Phi}^{\prime}$ & $\mathbf{P}$ & \multicolumn{1}{c}{ Autor } \\
\hline D. obesulus & Perú & 1.00 & 46.00 & 3.33 & 4.99 & Arntz et al.,(1987) \\
D. obesulus & Perú & 1.78 & 30.68 & 3.22 & 4.71 & Presente estudio \\
D. obesulus & Perú & 0.70 & 42.00 & 3.09 & 4.71 & Arntz et al.,(1987) \\
D. obesulus & Perú & 1.17 & 35.00 & 3.16 & 4.70 & Arntz et al.,(1987) \\
D. denticulatus & Venezuela & 1.79 & 30.02 & 3.21 & 4.69 & Marcano et al. (2003) \\
D. denticulatus & Venezuela & 1.48 & 31.47 & 3.17 & 4.66 & García et al. (2003) \\
D. hanleyanus & Argentina & 1.18 & 33.50 & 3.12 & 4.65 & Penchaszadeh \& Olivier (1975) \\
D. hanleyanus & Uruguay & 0.80 & 33.00 & 2.94 & 4.46 & Defeo (1996) \\
D. hanleyanus & Argentina & 0.79 & 34.13 & 2.96 & 4.50 & Luzzatto (2007) \\
D. hanleyanus & Brasil & 0.90 & 28.50 & 2.86 & 4.32 & Cardoso \& Veloso (2003) \\
D. striatus & Brasil & 1.16 & 25.10 & 2.86 & 4.26 & Rocha-Barreira et al. (2002) \\
D. dentifer & Costa Rica & 0.30 & 46.00 & 2.80 & 4.19 & Palacios et al. (1983) \\
D. dentifer & Colombia & 0.62 & 29.30 & 2.73 & 4.19 & Riascos \& Urban (2002) \\
D. hanleyanus & Brasil & 0.80 & 26.40 & 2.75 & 4.17 & Cardoso \& Veloso (2003) \\
\hline
\end{tabular}


Enero - Diciembre 2008

Tabla 3. Producción $\left(P, \mathrm{~g}\right.$ PSLC $\mathrm{m}^{-2}$ año $\left.{ }^{-1}\right)$, Biomasa $\left(\bar{B}, \mathrm{~g}\right.$ PSLC $\left.\mathrm{m}^{-2}\right)$ tasas de renovación $(P / \bar{B})$ en especies de género Donax en distintas partes del mundo.

\begin{tabular}{lcccccl}
\hline \multicolumn{1}{c}{ Especie } & Lat. & Long. & $\boldsymbol{P}$ & $\bar{B}$ & $\boldsymbol{P} / \boldsymbol{B}$ & \multicolumn{1}{c}{ Autor } \\
\hline D. obesulus & $12^{\circ} 50^{\prime} \mathrm{S}$ & $76^{\circ} 36^{\prime} \mathrm{W}$ & 3.76 & 3.14 & 1.20 & Presente estudio \\
D. hanleyanus & $23^{\circ} 03^{\prime} \mathrm{S}$ & $43^{\circ} 34^{\prime} \mathrm{W}$ & 3.67 & 2.53 & 1.45 & Cardoso \& Veloso (2003) \\
D. hanleyanus & $32^{\circ} 20^{\prime} \mathrm{S}$ & $52^{\circ} \mathrm{W}$ & 13.56 & 3.45 & 3.90 & Gianuca (1985) \\
D. serra & $22^{\circ} 47^{\prime} \mathrm{S}$ & $1^{\circ} 33^{\prime} \mathrm{E}$ & 356.60 & 288.80 & 1.20 & Laudien et al. (2003) \\
D. serra & $22^{\circ} 47^{\prime} \mathrm{S}$ & $1^{\circ} 33^{\prime} \mathrm{E}$ & 273.20 & 171.90 & 1.60 & Laudien et al. (2003) \\
D. serra & $22^{\circ} 5^{\prime} \mathrm{S}$ & $1^{\circ} 24^{\prime} \mathrm{E}$ & 637.30 & 545.90 & 1.20 & Laudien et al. (2003) \\
D. serra & $22^{\circ} 5^{\prime} \mathrm{S}$ & $1^{\circ} 24^{\prime} \mathrm{E}$ & 166.90 & 141.20 & 1.20 & Laudien et al. (2003) \\
D. sordidus & $33^{\circ} 57^{\prime} \mathrm{S}$ & $2^{\circ} 40^{\prime} \mathrm{E}$ & 0.38 & 0.21 & 1.78 & McLachlan \& van der Horst (1979) \\
D. sordidus & $33^{\circ} 57^{\prime} \mathrm{S}$ & $25^{\circ} 40^{\prime} \mathrm{E}$ & 0.15 & 0.10 & 1.56 & McLachlan (1979) \\
D. sordidus & $33^{\circ} 57^{\prime} \mathrm{S}$ & $25^{\circ} 40^{\prime} \mathrm{E}$ & 0.13 & 0.12 & 1.03 & McLachlan (1979) \\
D. trunculus & $45^{\circ} 50^{\prime} \mathrm{N}$ & $1^{\circ} 20^{\prime} \mathrm{W}$ & 18.98 & 15.42 & 1.23 & Ansell \& Lagardère (1980) \\
D. trunculus & $45^{\circ} 50^{\prime} \mathrm{N}$ & $1^{\circ} 20^{\prime} \mathrm{W}$ & 39.99 & 39.35 & 1.02 & Ansell \& Lagardère (1980) \\
D. vittatus & $45^{\circ} 50^{\prime} \mathrm{N}$ & $1^{\circ} 20^{\prime} \mathrm{W}$ & 12.33 & 7.66 & 1.61 & Ansell \& Lagardère (1980) \\
D. vittatus & $45^{\circ} 50^{\prime} \mathrm{N}$ & $1^{\circ} 20^{\prime} \mathrm{W}$ & 17.33 & 10.43 & 1.66 & Ansell \& Lagardère (1980) \\
D. vittatus & $51^{\circ} 41^{\prime} \mathrm{N}$ & $4^{\circ} 28^{\prime} \mathrm{W}$ & 0.60 & 0.29 & 2.10 & Warwick et al. (1978) \\
\hline
\end{tabular}

${ }^{1}$ Facultad de Pesquería, Universidad Nacional Agraria La Molina, La Molina, Lima, Perú. email: jah_arturo@hotmail.com 